\title{
A Wideband Microstrip Dipole Antenna Design for WLAN/WiMAX Applications
}

\author{
Adnan Sondas \\ Department of Information Systems Engineering, Kocaeli University, Kocaeli, Turkiye \\ *corresponding author, E-mail: asondas@kocaeli.edu.tr
}

\begin{abstract}
Recently, microstrip antennas are preferred in all areas of wireless communication, due to their advantages such as low volume coverage, light weight, surface compatibility, high cost requirements and easy production etc. The main disadvantage of these antennas is their narrow band performance $(\sim 11 \%)$. In the literature, there are some wideband microstrip antenna designs. These broadband characteristics are obtained by changing the antenna geometry or by adding new parasitic patches to the antenna elements. In this study, a classical wideband microstrip dipole antenna (MDA) design which can be used in WLAN/WiMAX applications (covering the bands 2.4-2.5 GHz and 2.5-3.5 GHz) is introduced. The proposed antenna has a pair of twisted strip which are placed asymmetrically near the feed of the dipole element with a length of $52 \mathrm{~mm}(\sim \lambda / 2)$. Also a pair of square loop elements is placed on a sublayer. The proposed MDA has a resonance between $2.06-3.72 \mathrm{GHz}$ with a bandwidth of $57 \%$. The antenna has a directive radiation pattern with a gain of 6.49-3.98 dBi.
\end{abstract}

\section{Introduction}

Microstrip antennas (MAs) are used frequently in wireless communication systems as they have many advantages such as low volume, low cost, lightweight and easy to manufacture. Also thanks to their low size, MAs can be easily inserted into or out of miniature-sized devices In addition, due to their surface compatibility, they can be mounted on the vehicles which is being required special precision such as airplanes, missiles and satellites without disturbing the aerodynamic structure of the vehicles. If necessary, the integrated antenna system obtained by placing the antenna on the same base material as the circuit elements provides a more ideal electrical performance. Furthermore, multi-band characteristics can be obtained from these antennas, while linear or circular polarized radiation characteristics can be obtained by small changes on the antenna design. [1].

In contrast to features mentioned above, MAs also have disadvantages such as low gain, low power and narrow-band performances $[2,3]$. Hence, the challenge in microstrip antenna design in wide/ multi -band implementation is to increase the bandwidth $(<\% 11)$. Studies in the literature, it is seen that the broadband limit, which is accepted as $20 \%$ and 25\% according to Defense Advanced Research Project Agency (DARPA) and Federal Communication Commission (FCC) respectively can be overcome for microstrip antennas [4]. When these studies in the literature are examined, it is seen that conventional MDAs are not preferred for these applications. Also, in these studies, it was observed that the wide band antenna performance could be achieved by the addition of different loadings $[5,6]$ to the dipole elements and the use of curved structures in the antenna design [7].

In this study, MDA design with broadband performance which is an alternative to the methods applied to increase the bandwidth of a classical dipole antenna (maximum 22\% performance) proposed in [6] has been introduced. The proposed MDA design is projected to be use in WiMAX $(2.4-2.5 \mathrm{GHz})$ and WLAN $(2.5-3.6 \mathrm{GHz})$ applications.

Note that this paper is an extended version of [8], which was previously presented at the URSI-Türkiye'2018 conference.

\section{Numerical design of the wideband antenna}

The proposed MDA design along with its design parameters are given in Figure 1. As seen, the proposed antenna design consists of a metallic ground plane and a double-layered radiating element. Top layer of the antenna is shown in Fig 1(a). In the top layer of the radiating element, two twisted loading elements attached asymmetrically to the feed point were added to the microstrip dipole antenna of $52 \mathrm{~mm}$ $(\sim \lambda / 2)$ length. These metallic elements are placed on a dielectric substrate (Arlon DiClad 880) with thickness of $0.79 \mathrm{~mm}$ and size of $60 \times 22 \mathrm{~mm}^{2}$. Also bottom layer of the antenna is shown in Fig 1(b). As seen, there are two metallic square ring elements on the substrate of the same dimensions. These rings are placed just below to the arms of the microstrip dipole in the upper layer. The positions of these rings are very critical for the desired antenna performance. In the CST Microwave Studio program, metallic elements were modeled using lossy copper plates $(\sigma=5.8 \times 107 \mathrm{~S} / \mathrm{m})$ of $0.05 \mathrm{~mm}$ thickness. In addition, a ground plane (GP) was placed at a distance of $30 \mathrm{~mm}$ so that the antenna radiation was directional. Modelled antenna's perspective view and the prototype of the antenna are shown in Fig. 1(c) and Fig. 1(d) respectively. 


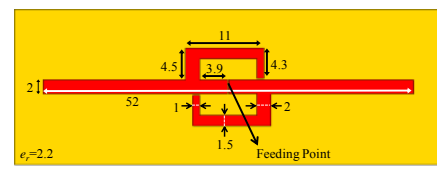

(a)

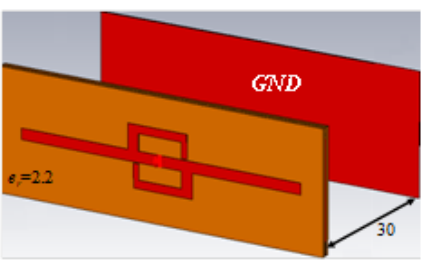

(c) (b)

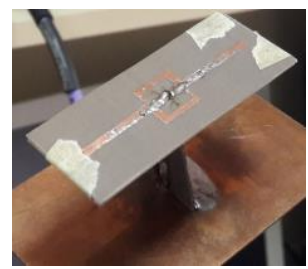

(d)

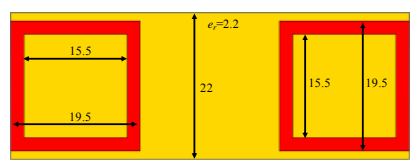

Figure 1: The proposed wideband MDA design a) top layer b) bottom layer c) perspective view d) fabricated (all dimensions are in $\mathrm{mm}$ ).

The input reflection coefficient $\left(\mathrm{S}_{11}\right)$ characteristics of the design steps are displayed in Figure 2. As can be seen, the $\lambda / 2$ dipole element provides a single-band operation around $2.4 \mathrm{GHz}$ with a bandwidth of $11 \%$. By placing the twisted strips asymmetrically near the feed of the dipole element, the antenna has a performance between $2.28-3.64 \mathrm{GHz}$ with a bandwidth of $46 \%$. Finally, by inclusion of the sublayer, the antenna has a resonance frequency between 2.06-3.72 $\mathrm{GHz}$ with a bandwidth of $57 \%$, covering designated WLAN/WiMAX bands. Note that $|\mathrm{S} 11| \leq-10 \mathrm{~dB}$ criterion with $50 \Omega$ system impedance is considered.

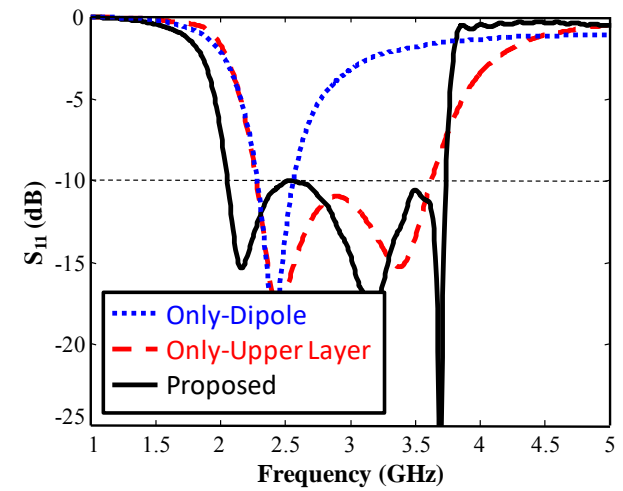

Figure 2: The simulated return loss characteristics of the design steps.

In addition, the computed VSWR characteristic of the proposed MDA is displayed in Fig. 3. As seen the antenna has a VSWR performance under level 2 between $2.06-3.72 \mathrm{GHz}$.

In Fig. 4, we display the simulated and measured return loss characteristics of the proposed MDA. As seen, the agreement between the results is quite good except that a little amount of bandwidth reduction is observed for the fabricated antenna. The discrepancies are probably due to material and fabrication tolerances. We note that the return loss measurements were carried out using Rohde \& Schwarz ZVB8 Vector Network Analyzer.

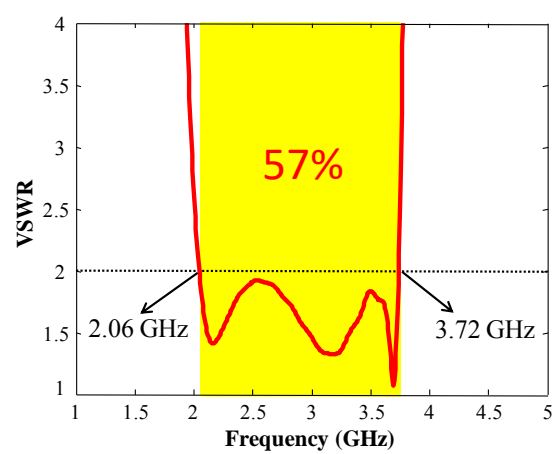

Figure 3: The simulated VSWR performance

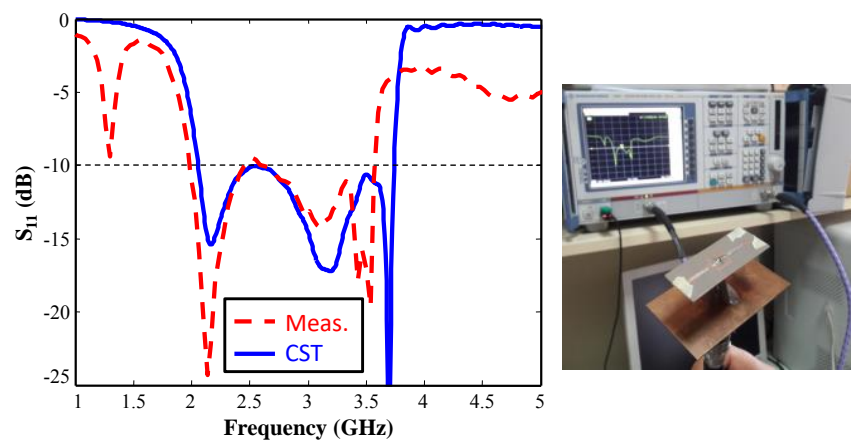

Figure 4: The return loss characteristics of the design: the CST simulation, and the measured result for the fabricated antenna are shown

The computed radiation patterns of the proposed MDA are shown in Fig. 5. As seen, the antenna has a directional radiation pattern performance whole band of the interest in both $\varphi=0^{\circ}$ and $\varphi=90^{\circ}$ planes.
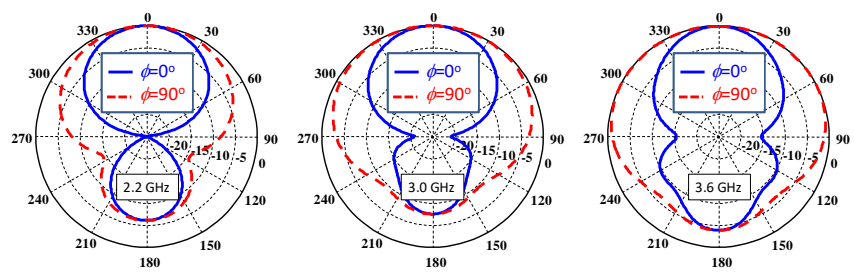

Figure 5: The computed radiation patterns of the antenna design at $2.2 \mathrm{GHz}$ (left), $3.0 \mathrm{GHz}$ (middle) and $3.6 \mathrm{GHz}$ (right).

In Fig. 6, we also display the computed surface current distributions over the antenna aperture for all the frequency bands. As seen, the current distribution at $2.2 \mathrm{GHz}$ is mainly concentrated along the dipole, while the distribution at $3.0 \mathrm{GHz}$ and $3.6 \mathrm{GHz}$ is predominantly occurs around the twisted strip elements. These results also confirm that the lower frequency band is mainly due to the dipole resonance, but the higher band occurs owing to presence of the strip elements. 

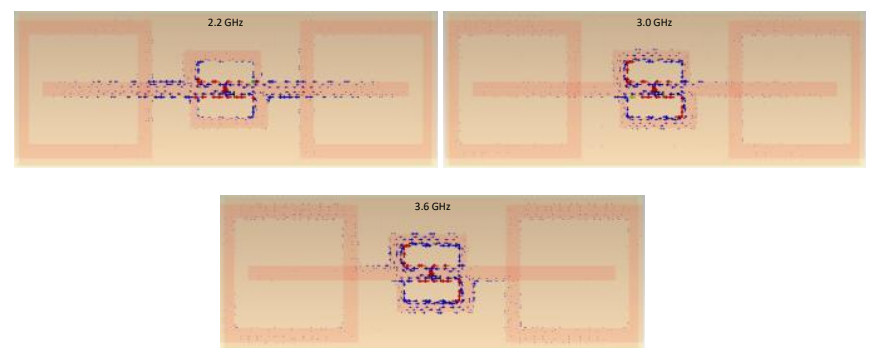

Figure 6: The surface current distributions over the antenna aperture at $2.2 \mathrm{GHz}$ (top-left), $3.0 \mathrm{GHz}$ (top-right) and $3.6 \mathrm{GHz}$ (bottom).

\section{Parametric studies}

In order to evaluate the performance of the proposed MDA, some parametric studies have been carried out to show the effects of the antenna parameters, namely the dipole length/width, parasitic element's length, ring dimensions. Below, we briefly discuss those studies.

\subsection{Dipole length/width}

The proposed MDA design consists of a pair of twisted strip placed asymmetrically near the feed of the dipole element with a length of $52 \mathrm{~mm}$ and a width of $2 \mathrm{~mm}$. As can be seen from Figure 7 (a), lower band of the $S_{11}$ characteristic is shifted by changing the length of the dipole. As expected, the resonance frequency shifts to lower values as the dipole length is extended. Also the dipole width effects only the $\mathrm{S}_{11}$ levels (see Fig. 7 (b)). By changing the length/width of the dipole element, desired impedance matching performance is disrupted.

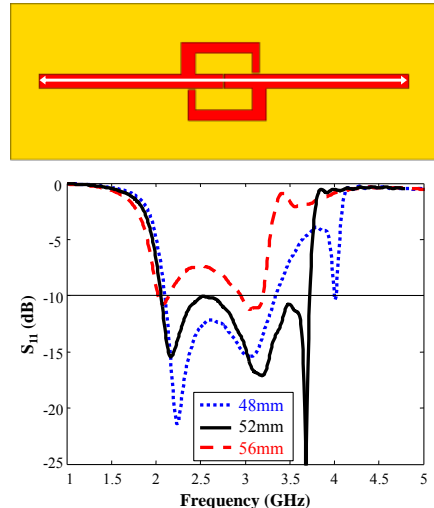

(a)
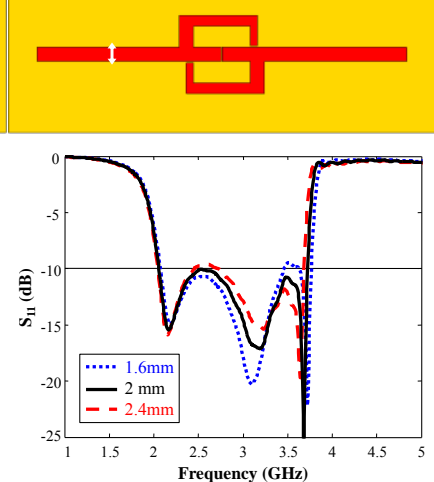

(b)

Figure 7: The effects of a) the dipole length b) the dipole width on the return loss performance.

\subsection{Parasitic elements' length}

Parasitic elements increase the band width performance of the MDA. As can be seen from Figure 8, the center band of the $S_{11}$ characteristic is shifted by varying the length of the $x$ and $y$ dimensions of the parasitic elements (see Fig. 8 (a) and Fig. 8 (b) respectively). By changing the $x$ and $y$ dimensions of the parasitic elements, desired impedance matching performance is also disrupted.

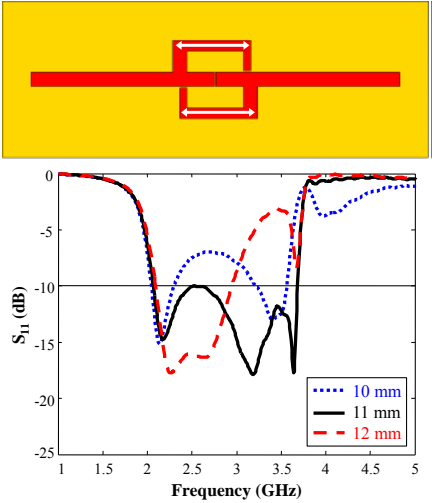

(a)
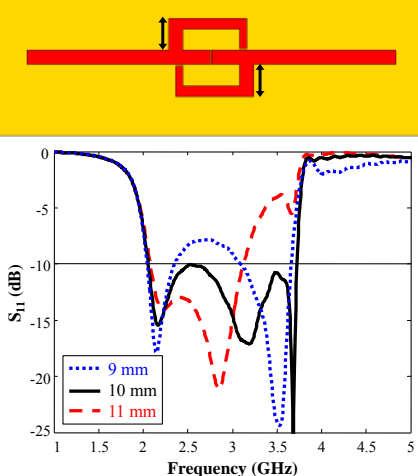

(b)
Figure 8: The effects of a) the dipole length b) the dipole width on the return loss performance.

\subsection{Ring dimensions}

As can be seen from Figure 9 (a), the middle and the upper bands of the $S_{11}$ characteristic shifts by changing the length of the ring elements' $x$ dimension. However, the $y$ dimension of the ring elements is only affects the upper band of the $S_{11}$ performance. (see Fig. 9 (b)).
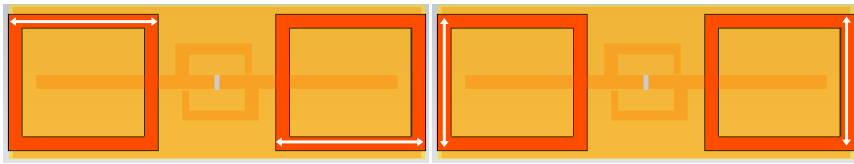

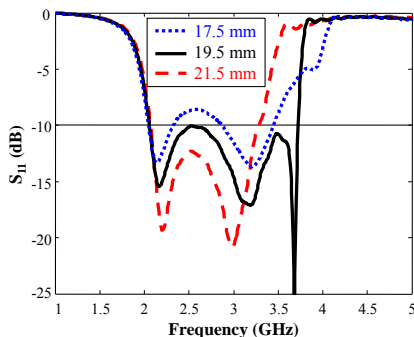

(a)

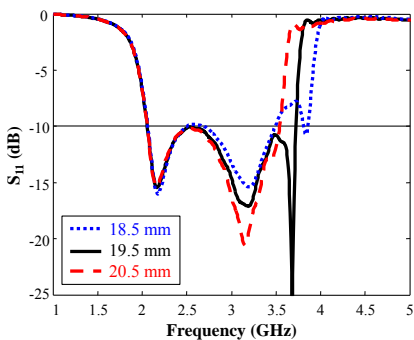

(b)
Figure 9: The effects of a) the ring elements' $x$ dimensions b) the ring elements $y$ dimensions on the return loss performance.

\section{Conclusions}

In this paper, we have introduced a novel wideband microstrip dipole antenna design with a bandwidth of $57 \%$ for WLAN/WiMAX applications in the $2.06-3.72 \mathrm{GHz}$ band. The proposed antenna has a pair of twisted strip which are placed asymmetrically near the feed of the dipole element with a length of $52 \mathrm{~mm}(\sim \lambda / 2)$. Also a pair of square loop elements is placed on a sublayer. The antenna has a directive radiation pattern with a gain of $6.49-3.98 \mathrm{dBi}$.

The proposed antenna was designed via the CST simulator, and the corresponding prototype was fabricated. It has been demonstrated that the simulations $\mathrm{S}_{11}$ performance agree quite well with the measurement except that a little amount of bandwidth reduction is observed. The discrepancies are probably due to material and fabrication 
tolerances. Also the effects of some critical design parameters on the antenna performance are examined.

\section{Acknowledgements}

The author would like to thank Dr. Mustafa H. B. UCAR and Dr. Yunus E. ERDEMLI for their invaluable comments.

\section{References}

[1] A. Sondaş, Metamateryal altyapılı ve halka yüklemeli mikroşerit anten tasarımları ve gerçeklenmesi, Kocaeli University Institute of Science and Technology, PhD Thesis, Kocaeli, Turkiye, 2011.

[2] G. Kumar, K.P. Ray, Broadband Microstrip Antennas, Artech House, USA, 2003.

[3] K. L. Wong, Compact and Broadband Microstrip Antennas, John Wiley \& Sons, 2002.

[4] FCC 02-48, Commission's Rules Regarding UltraWideband Transmission Systems, FCC, 98-153, Washington, 2002.

[5] S.L. Chen, K.H. Lin, Performance of a Folded Dipole with Closed Loop for RFID Applications, Progress In Electromagnetics Research Symposium, Prague, Czech Republic, 2007.

[6] N. Keskin, T. İmeci, S.M.T. Rahman, E. Karaçuha, UHF RFID Pasif Etiket için Dipol Anten Tasarımları, IEEE 22nd Signal Processing and Communications Applications Conference (SIU), Trabzon, Turkiye, 2014.

[7] K.V.S. Rao, P.V. Nikitin, S.F. Lam, Antenna design for UHF RFID tags: a review and a practical application, IEEE Trans. Antennas Propagation, 53:12, 3870-3876, 2005.

[8] A. Sondaş, Çift-Katmanlı Geniş-Bant Mikroşerit Dipol Anten Tasarımı, IX. URSI-Türkiye'2018 Bilimsel Kongresi, Konya, Turkiye, 2018. 